\title{
Identification of laccases involved in lignin polymerization and strategies to deregulate their expression in order to modify lignin content in Arabidopsis and poplar
}

\author{
Jouanin Lise ${ }^{1 *}$, Berthet Serge ${ }^{1}$, Mazel Julien', Demont-Caulet Nathalie ${ }^{1}$, Ayangma Brice $^{2}$, Le-Bris Philippe ${ }^{1}$, \\ Baratiny Davy', Leplé Jean Charles², Lapierre Catherine ${ }^{1}$
}

From IUFRO Tree Biotechnology Conference 2011: From Genomes to Integration and Delivery Arraial d'Ajuda, Bahia, Brazil. 26 June - 2 July 2011

Lignins have a major impact on the agro-industrial uses of plants. Until now, most of the strategies considered for lignin reduction have targeted the monolignol pathway since the genes involved in these metabolic steps have been identified in many plants. Less is known about the other steps and in particular on lignin polymerization in the cell wall. While it is established that peroxidases are involved in the polymerization of lignin precursors, it is not yet clear whether laccases (EC 1.10.3.2) participate in constitutive lignification.

In order to address this issue, laccase genes (AtLAC4 and AtLAC17) that are highly expressed in Arabidopsis stems were studied. AtLAC17 was specifically expressed in the interfascicular fibers while AtLAC4 was expressed in vascular bundles and interfascicular fibers. Arabidopsis T-DNA insertion mutants were selected and characterized. Two double mutants were obtained by crossing the AtLAC17 (lac17) mutant with two AtLAC4 mutants (lac4-1 and lac4-2). The single and double mutants displayed normal growth, except the lac4-2 lac17 mutant that sometimes had a semi-dwarf phenotype and collapsed vessels. While the single mutants had moderately reduced lignin levels, the stems of lac4-1 lac17 and lac42 lac17 had lignin content reduced by $20 \%$ and $40 \%$, respectively. This lower lignin level improved their saccharification yield. Thioacidolysis revealed that disrupting AtLAC17 mainly affected the deposition of G lignin units in the interfascicular fibers and that complementation of

\footnotetext{
* Correspondence: jouanin@versailles.inra.fr

'IJPB, UMR1318 INRA-AgroParisTech, 78026 Versailles, France Full list of author information is available at the end of the article
}

lac17 with AtLAC17 restored the normal lignin profile. This study provides evidence that both AtLAC4 and AtLAC17 contribute to the constitutive lignification of Arabidopsis stems and that AtLAC17 is involved in the deposition of $\mathrm{G}$ lignin units in fibers, suggesting a role in early lignification (Berthet et al, in press).

The double mutants cannot be obtained for species that are propagated vegetatively such as poplar. In order to produce plants with lower laccase activity and reduced lignin content, we therefore used a miRNA strategy. The overexpression of two miRNA (miR397 and miR408) targeting several laccase genes was tested in different plants including Arabidopsis and poplar. These miRNAs were expressed constitutively under the control of the CaMV $35 \mathrm{~S}$ promoter or of lignin-specific promoters such as $C A D$ and $C 4 L$ in transgenic Arabidopsis and poplar.

Results obtained using of this miRNA strategy in Arabidopsis and preliminary results for poplar will be presented.

\section{Author details \\ ${ }_{1}^{1}$ JPPB, UMR1318 INRA-AgroParisTech, 78026 Versailles, France. ${ }^{2} \mathrm{AGPF}$, INRA, 45002 Ardon, France.}

Published: 13 September 2011

doi:10.1186/1753-6561-5-S7-O39
Cite this article as: Lise et al.: Identification of laccases involved in lignin
polymerization and strategies to deregulate their expression in order to
modify lignin content in Arabidopsis and poplar. BMC Proceedings 2011
5(Suppl 7):O39.

( 2011 Lise et al; licensee BioMed Central Ltd. This is an open access article distributed under the terms of the Creative Commons 